\title{
A STUDY OF THREE RS CVn-TYPE ECLIPSING BINARIES
}

\author{
M.B.K. SARMA \\ Centre of Advanced Study in Astronomy, \\ Osmania University, \\ HYDERABAD-500 007, India.
}

I would like to present the results of the studies of three RS CVn-type eclipsing binaries that are conducted by our group at the Osmania University.

1. RZ Eridani is a member of the long period group of the RS CVn systems (Hall 1976). A photographic light curve of this system, published by Gaposchkin (1951), had indicated the primary eclipse to be total. The radial velocity curve published by Cesco and Sahade (1945) had indicated an eccentric orbit with $e=0.36$ and $f(m)=0.060$. We observed this system photoelectrically in UBV colours using the $1.2 \mathrm{~m}$ reflecting telescope of the Japal-Rangapur observatory during 1976-79 and the following improved ephemeris was derived:

$$
\text { Prim.Min }=\text { HJD } 2443888.3618+39^{d} .282596 E \text {. }
$$

As one full light curve could not be obtained in any one of the years, the combined light curve was used for analysis. The light curves in the three colours showed small distortion suggesting the presence of stellar spots. The primary eclipse is occultation (total) and the secondary eclipse is not detected. Preliminary analysis has indicated the system to consist of a hotter and smaller F6 V and cooler and bigger K2 IV components. Assuming the $F 6 \mathrm{~V}$ component to be normal having a radius of $1.17 \mathrm{R}_{\odot}$ and mass of $1.25 \mathrm{~m} \odot$ and using the radial velocity data of Cesco \& Sahade, a radius of $3.15 \mathrm{R}_{\odot}$ and mass of $0.59 \mathrm{~m}_{\odot}$ are derived for the $\mathrm{K} 2 \mathrm{IV}$ component. A temperature of $4590^{\circ} \mathrm{K}$ and radius of $3.15 \mathrm{R}_{\odot}$ give a bolometric magnitude of 3.25 for the cooler component. From the mass - luminosity relation for normal stars, a star of mass $0.59 \mathrm{~m}_{\odot}$ will have a bolometric magnitude of 6.88 (Allen 1973). From this it is inferred that the cooler component is overluminous for its mass by about 4 magnitudes. In this respect it resembles the secondaries of Algol systems. We ther efore conclude that the cooler and less massive K2 IV component is in an advanced state of Post-main-sequence evolution. The final analysis is in progress. Spectroscopic elements of this system are urgently needed for an understanding of the evolutionary status of the components. 
2. The eclipsing binary GK Hydrae was classified as regular member of the RS CVn group of stars by Hall (1976). A photographic light curve of this system was published by Strohmeier and Knigge (1962) and an incomplete photoelectric light curve by Popper and Dumont (1977). The available spectroscopic data on this system is very scanty.

We have observed this system during 1977-1983 and the following improved ephemeris was obtained:

$$
\text { Prim.Min }=\text { HJD } 2445376.1318+3^{\mathrm{d}} .58703633 \mathrm{E} \text {. }
$$

Preliminary analysis (colours as well as depths of the eclipses) has indicated that this system consists of a hotter and smaller F8 V star and a cooler, bigger and brighter G8 IV star. The smaller star is eclipsed at the deeper minimum. In this respect, it resembles SZ Psc (Jakate et al.1976). Assuming the masses of these components to be $1.2 \mathrm{~m}_{\odot}$ and $1.3 \mathrm{~m}_{\odot}$ (Popper 1982), it is found that these components are well within their Roche lobes, thus making it a detached system. The F8 V component with a radius of $1.3 \mathrm{R}_{\odot}$ and $\mathrm{M}_{\text {bol }}=3.8$ seems to have evolved slightly away from the zero age main-sequence. From the appearance of the light curves at the primary eclipse (1st and 4th contacts), it is concluded that the brighter G8 IV component ( $R=2.8 R_{\odot}$ and $\left.M_{b o l}=3.0\right)$ has an extended atmosphere. Due to its effect on the light curve, the distortion wave, if any, caused by spots, could not be detected. The final analysis is in progress. Radial velocity data for this system is urgently needed for understanding the nature of its evolution.

3. SV Camelopardalis is a short period RS CVn-type (BY Draconis ?) eclipsing binary for which Patkos (1982) has published extensive photoelectric observations. We have analysed this data for studying the nature of spot activity and obtain photometric elements. The presence of a third body in this system was discussed by Friboes-Conde and Herczeg (1973), Hilditch et al. (1979) and Busso et al. (1985). Taking all the available times of minima and using least square method, the ephemeris:

$$
\text { Pri.Min }=\text { HJD } 2442634.3520+0^{d} .59307141 \mathrm{E}
$$

is derived. With this ephemeris, the (O-C)s are obtained and applying Irwin's (1952) method, the elements of the light-time orbit are derived. They are: $\mathrm{P}=54.23$ years, $\mathrm{K}=0^{\mathrm{d}} .0079$, $\mathrm{e}=0.587 \pm 0.027, \omega=174.76^{\circ} \pm 2.4^{\circ}$ and $T$ (time of periastron passage) = HJD 2421283.7716. Using these elements, the effect of the 3rd body motion on all the observations of Patkos is removed. 10 good light curves are available for analysis. The light outside the eclipses is Fourier analysed up to $n=4$. Theoretical reflection and ellipticity coefficients are derived using Merrill's (1970) equations. From these Fourier and theoretical coefficients the wave coefficients are derived for the 10 light curves. The method of analysis is the same as that reported earlier by Vivekananda Rao and Sarma (1983). We could identify three wave minimia, two of which have direct motion with periods of 2 and 1.5 years while the third one has a retrograde motion with a 3.5 years period. If these three minima are identified with threepot groups, 
then, two of them having direct motion will be lying above the co-rotating latitude and the other having retrograde motion will be lying below the co-rotating latitude. From the percentage of light contributed by the wave amplitudes with respect to the luminosity of the hotter $(\mathrm{G} 2-3 \mathrm{~V})$ and cooler $(\mathrm{K} 4 \mathrm{~V})$ components, we could conclude that the hotter and more massive component of the system is the source of the distortion wave.

As SV Camelopardalis is a short period RS CVn-type binary, this result is in agreement with that reported by Vivekananda Rao and Sarma (1983) for UV Piscium.

After removing the effect of distortion wave from the observed light curves, we have expected them to merge together (for each colour) to give a unique solution. Even though the wave removal has made the brightness at the two elongations to be the same for each light curve, the 10 light curves have merged into three different groups with different brightnesses at elongations which we have termed as brighter, normal and fainter. In yellow, the difference between the brightness at elongations of the brighter and fainter groups is about $0^{m} \cdot 1$. From the study of the colour $(\Delta(B-V)$ and $\Delta(U-B)$ ) variations with phase in these three groups, we could conclude that the hotter component, which is in front at the time of the secondary eclipse (total) is the source of this variation. Thus we find that in SV Cam, the hotter star is not only having spots but is also a variable. This phenomenon is common to some of the RS CVn binaries eg. WY Cancri (Vivekananda Rao and Sarma (1983)). We are now solving the light curves in the three groups separately to find whether the derived elements will give a clue to the type of variation of the hotter component.

\section{REFERENCES}

Allen, C.W.: 1973, Astrophysical Quantities 3rd ed (Athlone Press) P 204. Busso, M., Scaltriti, F. and Cellino, A.: 1985, Astron. Astrophys.148, 29. Cesco, Carlos U. and Sahade, Jorge.: 1945, Astrophys.J.101, 370.

Friboes-Conde, H. and Herczeg, T.: 1973, Astr.Astrophys.Suppl.Ser. 12,1. Gaposchkin, Sergei.: 1951, Bull.Harv.Coll.Obs.No.920, 22.

Hall, D.S.: 1976, 'Multiple Periodic Variable Stars', (ed. W.S. Fitch), Part I, IAU Colloq. 29, 287.

Hilditch, R.W., Harland, D.M. and Mclean, B.J.: 1979, M.N.R.A.S. 187, 797. Irwin, J.B.: 1952, Astrophys.J.116, 211.

Jakate, S.M., Bakos, G.A., F ernie, J.D. and Heard, J.F.: 1976, Astron.J.81,250.

Merrill, J.E.: 1970, Vistas in Astronomy, 12, 43.

Patkos, L.: 1982, Communications. Konkaly Obs. No.80.

Popper, D.M. and Dumont, P.J.: 1977, Astron.J.82, 216.

Popper, D.M.: 1982, P.A.S.P., 94 , 945.

Strohmeier, W. and Knigge, R.: 1962, AN 286, 136.

Vivekananda Rao, P. and Sarma, M.B.K.: 1983, J.Astrophys.Astr. 4, 161. Vivekananda Rao, P. and Sarma, M.B.K.: 1983, 'Activity in Red-Dwarf Stars' (ed. P.B.Byrne and M. Rodono) P 399, Reidel Publishing Co. 